\title{
Artelogie
}

Recherche sur les arts, le patrimoine et la littérature de l'Amérique latine

5 | 2013

Femmes créatrices en Amérique latine : le défi de synthétiser sans singulariser

\section{Insurgée en tenure de noble : Lina Bo Bardi et l'architecture brésilienne de l'après guerre}

Zeuler R. M. de A. Lima

\section{OpenEdition}

Journals

Édition électronique

URL : https://journals.openedition.org/artelogie/5467

DOI : $10.4000 /$ artelogie. 5467

ISSN : 2115-6395

Éditeur

Association ESCAL

Référence électronique

Zeuler R. M. de A. Lima, «Insurgée en tenure de noble : Lina Bo Bardi et l'architecture brésilienne de l'après guerre », Artelogie [En ligne], 5 | 2013, mis en ligne le 16 octobre 2013, consulté le 20 décembre 2021. URL : http://journals.openedition.org/artelogie/5467 ; DOI : https://doi.org/10.4000/artelogie. 5467

Ce document a été généré automatiquement le 20 décembre 2021.

Association ESCAL 


\title{
Insurgée en tenure de noble : Lina Bo Bardi et l'architecture brésilienne de l'après guerre
}

\author{
Zeuler R. M. de A. Lima
}

\section{Introduction}

1 Malgré ses intenses activités, l'architecte brésilienne d'origine italienne Lina Bo Bardi (1914-1992) ne reçut pas pleine reconnaissance pour son travail pendant la plus part de sa vie. Pourtant, depuis sa mort, elle gagne de plus en plus l'attention des chercheurs et des designers, intéressés par sa contribution inestimable à l'architecture du $\mathrm{XX}^{\mathrm{e}}$ siècle. Souvent, des images de ses projets circulent dans des publications culturelles et professionnelles, présentées comme évidence d'une carrière sans parangonne et d'une œuvre intellectuelle et artistique originale émergée d'une trajectoire prolifique et d'une pensée influente, mais pas encore complètement comprise.

2 Bo Bardi occupa, dans sa jeunesse, une position unique parmi d'autres architectes en Italie et, en particulier dans sa maturité, au Brésil : d'abord, une femme et, après, une femme étrangère. Malgré - ou peut-être à cause de - telles circonstances, elle eut accès à des milieux culturels très actifs, qui contribuèrent à ses réalisations, mais également à ses défis. Elle gagna accès à la culture dominante et, au même temps, elle chercha de la mettre en question. Sa production inclut tant des travaux d'illustration et d'édition que des projets d'architecture et d'exposition, développés dans le mouvement entre des différentes conditions sociales et des institutions culturelles aussi bien qu'entre deux pays et, surtout, entre différentes villes : Rome et Milan, en Italie et São Paulo, Salvador et, dans une moindre mesure, Rio de Janeiro, au Brésil.

Dans les deux pays qu'elle habita, Bo Bardi témoigna des moments importants de la modernisation économique et culturelle marquée par fortes tensions politiques et réalités sociales en conflit. Dans ces situations et villes uniques, elle rassembla des ressources riches et variées qui ont abouti à des œuvres éphémères, expérimentales et 
emblématiques. Ses expériences intellectuelles et personnelles se communiquent avec le caractère prédominant hybride de son travail, aidant à étendre la compréhension de la négociation des valeurs personnelles, de la modernité en architecture et de leur sédimentation dans des conditions historiques et géographiques spécifiques, dans le cas du Brésil.

\section{Devenir un architecte au féminin}

Doté d'un tempérament perspicace, irrévérencieux et résolue - mais aussi sombre et mélancolique, Bo Bardi ne pourrait pas avoir prédit dans sa jeunesse qu'elle deviendrait une personnalité influente, particulièrement si l'on considère qu'elle acquit reconnaissance en dehors de son pays natal et dans un domaine traditionnellement contrôlé par une élite masculine. Pourtant, à la fin de sa vie, elle déclara, « au Brésil,j'ai toujours fait tout ce que je voulais. » Elle renforça son avis en ajoutant, «Je n'ai jamais fait face à des obstacles, même pas comme une femme. C'est pour cela que je dis que je suis stalinienne et antiféministe.»(BO BARDI, 1989a:5). Elle n'expliqua pas, pourtant, ce qu'elle voulait dire avec de pareilles assertions provocatrices, qui furent publiées par des journalistes, contribuant à sa mythologie personnelle. Bo Bardi ne voyait pas Staline comme un dirigeant politique douteux, mais comme un héros dans la libération de l'Italie du Nazi-fascisme. En outre, elle ne expliquait pas que ce qu'elle opposait du féminisme était le mouvement de libération de la femme, qui, pour toutes ses luttes juridiques, elle considérait comme une contestation bourgeoise. En effet, elle admirait la génération précédente qui s'avait battu pour l'égalité de droits, ajoutant que, parmi les classes les plus pauvres, les femmes s'efforçaient toujours à côté des hommes.

Née à Rome dans une famille de ressources financières limitées, peu de temps avant l'entrée de l'Italie à la Première Guerre Mondiale, Bo Bardi grandit au cours de la montée du fascisme et arriva à la vie professionnelle à l'exorde de la Seconde Guerre Mondiale. Sa jeunesse fut entourée par de graves problèmes économiques et politiques, et elle fut élevée dans une culture où la plupart des femmes, en particulier parmi les familles à basse revenu, étaient limitées à des rôles sociaux soumis. De plus, le Fascisme renforça cette condition à travers son modèle moderne et ambigu de la femme-mère (domestique, forte et productive) contre la femme-crise (courtoise, décadente et stérile) (DE GRAZIA, 1993 :57). Sur le plan professionnel, notamment dans le domaine en plein essor de l'architecture, la division du travail n'était pas différente et présentait des grands désavantages aux femmes. Malgré la crise sociale et la tension émotionnelle de ces années, Bo Bardi ne se laissa pas intimider, défiant les conventions, et s'adaptant aux situations changeantes pour accomplir ses ambitions.

Alors qu'elle grandit se méfiant des rôles sociaux féminins conventionnels, elle se pencha à l'opposé de la présence stricte et traditionnelle de sa mère et vers les plus tolérantes et passionnantes figures masculines dans sa famille. Par exemple, son oncle maternel, un journaliste et auteur-compositeur avec des convictions fascistes mais aussi ami d'artistes futuristes, présenta la jeune Lina à la culture populaire du théâtre et du cinéma. Ces spectacles sans prétention poussèrent sa curiosité et façonnèrent son jeune répertoire esthétique. Surtout son père, Enrico Bo, un constructeur et peintre amateur donna à leur foyer un certain anarchisme et il fut particulièrement favorable aux intérêts artistiques de sa fille ainée, en l'enseignant les principes de dessin, de 
perspective, et l'utilisation des couleurs en aquarelle, qu'elle développa plus tard dans le Lycée d'Art à via Ripetta.

7 Son éducation secondaire et son éducation professionnelle dans la Ecole d'Architecture de Rome l'exposèrent à des valeurs historiques et humanistiques et à des principes de composition académiques qu'elle critiqua du point de vue rhétorique, mais qu'elle ne réfuta pas esthétiquement, même en ayant fortement embrassé des principes modernes au long de sa vie. Malgré ses réserves sur sa ville natale, surtout après sa conversion au rationalisme à Milan pendant la Seconde Guerre Mondiale, elle n'hésita pas à dire, à la fin de sa vie, que « Rome fut une grande école d'architecture » (BO BARDI, 1989b : 1).

Le fait qu'il n'y avait, dans son école d'architecture, que quelques femmes parmi les 120 élèves - et aucune enseignante - ne semble pas avoir dérangé Lina Bo Bardi. Elle était parfois à l'écart, mais elle n'était pas timide. Elle ne parlait jamais de sa vie romantique mais mentionna aimer le fait que certains de ses camarades de classe aimaient flirter avec elle (TENTORI, 1995: 4). Son observation corrobore un fait de sa jeunesse confirmé par ceux qui l'ont bien connue tout au long de sa vie - qu'elle était au courant de ses séduisants attributs féminins lorsqu'elle se familiarisa avec des hommes influents dans le milieu architectural italien. Avec son premier petit ami, son bien nanti collègue Carlo Pagani, elle entra dans la modernité culturelle et professionnelle de Milan au début de la Seconde Guerre Mondiale, mais il fut avec Pietro Maria Bardi, le marchand d'art romain, le polémique journaliste et promoteur de l'art moderne et de l'architecture rationaliste italienne, qu'elle décida de traverser l'océan Atlantique après la fin du conflit.

\section{Architecture en papier}

Malgré la guerre, Milan offrit un important contrepoint à l'éducation romaine classique de Bo Bardi. Pagani, qui enseignait à l'Institute Polytechnique et collaborait avec Gio Ponti, ouvrit les portes des opportunités professionnelles qu'elle eut au cours de son séjour de six ans en Lombardie. Même si elle ne travailla pas dans le bureau de Ponti, comme elle le propose dans ses notes biographiques, Bo Bardi collabora comme associée sur les attributions de projet graphique que l'éminent architecte -qui avait grande influence sur les cercles professionnels et éditoriaux milanais -remettait à Pagani. Les efforts de Ponti pour documenter l'artisanat italien, afin de l'associer à la production industrielle et de promouvoir la continuité entre tradition et modernité dans la maison italienne, devinrent des références conceptuelles significatives et permanentes pour la jeune architecte romaine.

Dans leur agence improvisée, Pagani contrôla souvent les contacts professionnels et les tâches quotidiennes, renforçant la division traditionnelle du travail entre hommes et femmes. Il profita de l'exposition publique tandis que Bo Bardi travailla dans la sphère privée du bureau. Ces contraintes, toutefois, ne limitèrent pas ses ambitions. En effet, elle saisit l'occasion pour développer ses compétences visuelles et l'écriture et, par la suite, quand Pagani fut envoyé pour lutter contre la guerre en Corse en 1943, elle prit contrôle de leur travail ensemble. Elle initialement trouva quelque difficulté pour affirmer son indépendance professionnelle tant que femme dans une nouvelle ville, mais elle ne tarda pas à se faire connaître.

11 Au delà de ces différences personnelles, elle faisait aussi face à l'adversité de devenir architecte, comme elle se souvenait, dans un moment où « rien n'était construit, et tout 
était détruit " (BO BARDI, 1989b :1). Son alternative fut de travailler dans le domaine éditorial et des illustrations. Ainsi, elle développa une expérience professionnelle considérable à travers des plusieurs contributions pour publications directement et indirectement contrôlées par Gio Ponti, tels que Lo Stile et Domus - tous deux dédiées à l'architecture - et magazines répondants à un public féminin comme Linea, Bellezza, Grazia et Cordelia entre 1941 et 1943. Elle eut aussi l'occasion d'apparaître tant qu'auteur et d'exprimer ses premières idées en architecture, comme dans sa première signature solo dans la recension du livre de l'architecte fonctionnaliste allemand Otto Völckers, Das Grundrisswerk (Travaux de Fondation). Dans son court texte, elle annonça surtout que "l'architecture est l'expression de la vie humaine, et elle porte un contenu moral profond ", un point clef qu'elle répéta plusieurs fois dans sa longue carrière (BO BARDI, 1941 : 34).

Fig. 1. Exemple d'illustrations et d'édition préparés par Lina Bo Bardi avec Carlo Pagani pour les pages du magazine Lo Stile, dirigé par Gio Ponti au début des années 1940

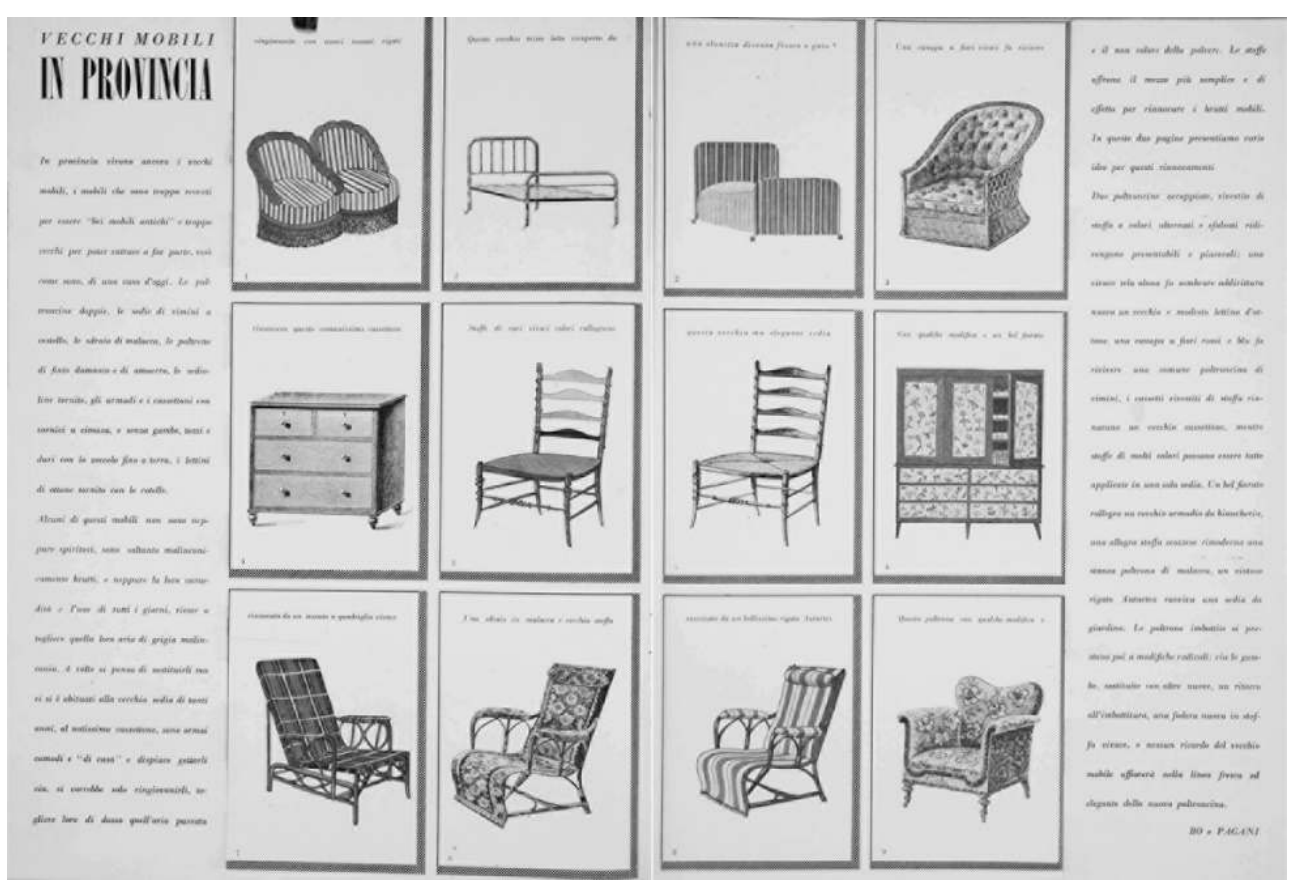

Collection Zeuler R. Lima

Même si Bo Bardi parfois se plaignait de la futilité de ses travaux pour les magazines de mode et décoration au cours de la guerre, le fait d'être une femme lui permit d'occuper un poste de commandement à Domus tandis que Pagani était au service militaire. Préoccupé d'être exposé à des opérations de ratissage constantes par les troupes nazies dans les voyages en train entre Milan et Bergame, où les bureaux d'impression du magazine avaient été relogés, Pagani suggéra que, en tant que femme, Bo Bardi serait mieux adaptée au va-et-vient entre les deux villes sans soulever des soupçons concernant le type d'information qu'elle pourrait transporter. Dans ces circonstances, elle devint coéditrice de Domus avec Pagani tout au long de 1944. Au delà de continuer la ligne éditoriale héritée de Ponti, ils lancèrent une ligne indépendante, les Quaderni di Domus, mettant en évidence des sujets contemporains tels que le logement, les besoins humains et la culture quotidienne. Par exemple, il publièrent le livre La maison de l'homme par Le Corbusier et François de Pierrefeu et un dossier de guerre titré «Centri di attesa par 'senza tetto'" (Refuges pour sans-abri) (LIMA, 2013: 34). Leur travail 
éditorial pour Domus fut de courte durée, mais il fut aussi son initiation dans le genre de sensibilités culturels et politiques qui fleuriraient au Brésil dans son âge mûr.

Dès que la fin de la guerre semblait proche, Bo Bardi et Pagani commencèrent à réfléchir sur la création d'un magazine économique portant sur des questions contemporaines italiennes et réunirent un petit nombre de jeunes architectes milanais pour discuter le future du pays. Ils baptisèrent leur groupe Organizzazione architetti associati (Organisation des architectes associés) et, juste avant que l'Italie capitule devant les forces alliées le 1er mai 1945, leur assemblée informelle élargit à quinze architectes, et ils fondèrent le MSA, Movimento studi architettura (Mouvement des études architecturales) (LIMA, $2013:$ 40).

La Seconde Guerre Mondiale prit fin, mais la situation sociale en Italie était catastrophique. Plusieurs architectes s'organisèrent, prévoyant un effort collectif vers l'urgence de la reconstruction. Et donc, Bo Bardi et Pagani aidèrent à organiser un grand rencontre professionnel et multidisciplinaire entre le 14 et 16 décembre, 1945. Le discours dramatique d'ouverture par Nathan Rogers mena une longue liste de présentations sur des sujets allant de l'emploi de technologies constructives traditionnelles et nouvelles aux modèles d'aménagement urbain. Parmi les conférenciers était Lina Bo, la seule femme à faire un discours public officiel, intitulé «La propaganda per la ricostruzione » (La publicité pour la reconstruction). Elle parla brièvement mais de manière incisive sur le rôle de la presse au service des causes publiques, anticipant une publication qu'elle et Pagani projetaient avec Bruno Zevi.

Ils imaginaient une revue offrant un forum pour le débat entre architectes, journalistes, écrivains et artistes, "réglant toutes les questions concernant l'architecture, » et annonçant une perspective que Bo Bardi maintiendrait tout au long de sa carrière : "l'architecture comme 'vie' » (ZEVI, $1945: 2$ ). C'est elle qui suggéra que le magazine serait simplement nommé A. Malgré son enthousiasme initial, sa collaboration avec le MSA et le magazine - plus tard renommé A, Cultura della Vita-diminua à mesure qu'elle commença à passer plus de temps à Rome, où Pietro Maria Bardi, avec qui elle avait commencé un rapport affectif plus proche, avait ouvert une nouvelle galerie d'art. La publication fut annulée en juin 1946 après neuf éditions, mais elle n'oublia jamais les leçons politiques et culturelles qu'elle en apprit.

16 Alors qu'elle travaillait pour le magazine, Bo Bardi produisit aussi son dernier projet en Italie. Pagani organisa un grand salon du meuble économique pour la Riunione italiana per le mostre di arredamento (RIMA, groupe italien pour les expositions de l'ameublement) dans l'été de 1946, et il invita son amie pour concevoir un stand pour des nouveaux matériaux textiles industriels. La salle inclut le travail de trois femmes: l'architecte Lina Bo, qui conçut un décor hybride de références industrielles et vernaculaires, et les designers du textile Gegia Bronzini et Fede Cheti, qui recherchaient et produisaient des tissus modernes basés sur des techniques traditionnelles.

17 Après ce projet, la collaboration professionnelle de Bo Bardi avec Pagani prit fin et elle déménagea à Rome vers la promesse d'une vie plus captivante en compagnie de Pietro Maria Bardi, qui examinait la possibilité d'étendre ses affaires commerciaux à l'étranger. Depuis sa première visite au Brésil et en Argentine en 1934, sont cible était l'Amérique du Sud, où les nouvelles élites capitalistes seraient disposés à investir au patronage artistique. Il avait fait la connaissance de quelques intellectuels reliés à l'ambassade du Brésil à Rome et, après la fin de la guerre, il apprit d'un journaliste 
brésilien excentrique, rusé et entrepreneur - le baron de presse Francisco de Assis Chateaubriand Bandeira de Mello - qui aspirait à créer un musée d'art selon le modèle des philanthropes nord-américains. Avec l'aide de l'ambassade du Brésil, Bardi organisa trois expositions d'œuvres de sa galerie à Rio de Janeiro entre décembre 1946 et mars 1947. Deux d'entre elles seraient organisées dans le nouveau bâtiment emblématique du Ministère de l'Education et de la Santé (MES) à Rio de Janeiro, conçu par Lucio Costa et de son équipe en dialogue avec Le Corbusier.

Plus qu'un partenaire romantique, Bardi (quatorze ans plus âgé que Bo Bardi) représentait une association symbolique avec l'histoire récente du modernisme en Italie et avec un homme fascinant et bien établi dont la compagnie offrait un future stimulant. D'un côté, elle admirait l'intelligence, l'astuce et l'audace de Pietro et prenait plaisir à séduire une figure publique si importante. D'un autre côté, Il se sentait flatté par la vigueur physique juvénile de Lina et attiré par son ambition et son attitude capricieuse. Le 24 septembre 1946, un mois après leur hâtive mariage, ils montèrent, au port de Naples, à bord de l'Almirante Jaceguay, un petit mais luxueux paquebot brésilien et se dirigèrent vers Recife et leur destination finale, Rio de Janeiro, avec une grande collection de peintures et d'objets d'art.

\section{Une entreprise commerciale devenue épopée culturelle}

19 Lina Bo Bardi arriva au Brésil à un moment propice. L'entreprise de son mari rencontra un environnement culturel aussi favorable que familier. Depuis les années 1930, Rio de Janeiro, alors que la capitale fédérale, était devenu l'épicentre du mouvement moderniste brésilien (commencé dix ans auparavant à São Paulo) avec la construction du MES comme sa vedette. Dans ses notes biographiques, Bo Bardi décrivit sa première vision du bâtiment comme "un grand navire blanc et bleu contre le ciel " (BO BARDI, $1989 \mathrm{~b}:$ 2). Conçu et construit entre 1936 et 1945, l'ensemble représenta les efforts des architectes brésiliens pour consolider l'architecture moderne dans le pays et pour associer cette architecture avec le projet de construction d'une nation moderne. Bo Bardi rétrospectivement le compara au "premier message de la paix à notre génération après le déluge de la Seconde Guerre Mondiale " (BO BARDI, 1989b : 1). Une autre fois, ditelle, «je me suis senti dans un pays inimaginable, où tout était possible. Je me sentais heureuse, et Rio n'avait pas des ruines » (BO BARDI, 1993 : vidéo).

Contrairement à Rome et Milan ou même Recife, Rio de Janeiro n'avait pas connu les atrocités de la guerre. Pourtant, le Brésil, comme Bo Bardi comprendrait plus tard, partageait certaines des caractéristiques sociales, culturelles et économiques de l'Italie. Comme son pays natal, le Brésil était une nation jeune formée au XIX ${ }^{\mathrm{e}}$ siècle et périphérique au système capitaliste; son économie reposait essentiellement sur la production agricole; et le pays était polarisé politiquement. Avant tout, les deux pays avaient vécu des remarquables processus de modernisation architecturale et artistique et entre les années 1930 et 1940 . Cependant, bientôt les développements d'après-guerre dans les deux pays prendrait sens distinctifs.

21 Le départ de Lina Bo Bardi d'Italie coïncida avec la période de confrontation, dans le domaine culturel, de la foi rationaliste par l'existentialisme néo-réaliste. Ce contexte produisit les semences d'un système de principes qu'elle transplanta sur le sol fertile, 
quoique difficile à cultiver, qu'elle rencontra avec l'architecture et le design brésilien. Elle tenait le projet moderniste au Brésil comme le résultat du rapport entre ses différentes identités culturelles et sociales. Malgré sa position sociale privilégiée, Bo Bardi développa des critères de modernité et d'identité à l'encontre de ceux que préconisaient les élites brésiliennes. Au long de ce processus, elle invertit doublement les valeurs de la modernité architecturale européenne: en premier lieu, par son expérience des marges, dans une Italie décentrée en Europe, et ensuite par la réalité qu'elle rencontra dans les marges, au Brésil.

Bien qu'elle et Bardi n'avaient pas initialement prévu de rester au Brésil, ils virent bientôt cette possibilité se concrétiser. Bardi, ayant le projet de vendre sa collection d'art, réussit à organiser ses expositions avec grand succès et à établir des affaires avec Assis Chateaubriand, qui, à la suite, l'invita pour concevoir et diriger son musée d'art. Bo Bardi suivit son mari, plongeant initialement (dans leur première décennie au Brésil) à l'ombre de son entreprise commerciale et artistique. ils discutèrent la création du musée d'art à Rio de Janeiro avec Chateaubriand, et Bo Bardi prépara un projet préliminaire pour un petit musée-galerie innovateur au centre-ville - son premier projet au Brésil. Son étude fut abandonné après que Chateaubriand décida d'accueillir le nouveau musée dans le bâtiment qu'il avait faire construire à São Paulo pour sa société de presse et qui accueillerait à partir d'Octobre 1947 le Musée d'Art de São Paulo (MASP).

23 Avant de quitter Rio de Janeiro, Bo Bardi développa grand intérêt pour la ville pendant son séjour de plus de six mois, et elle ne fut pas seulement impressionnée par son architecture moderne. Au-delà de ses réflexions sur l'édifice de Lucio Costa et de ses associés, sa curiosité fut attirée par la spontanéité et le manque de politesse qu'elle voyait dans la foule des rues de Rio de Janeiro. Pour elle, leur comportement était autant le signe de la privation d'éducation que celui de la transgression vivifiante des excès, et par là des formalités de la société bourgeoise européenne. Dans ses commentaires, elle disait que Rio de Janeiro était une ville cosmopolite qui attirait de nombreux étrangers, mais que, dans la confrontation avec le quotidien au Brésil, il ne pouvait y avoir de moyen terme (BO BARDI, 1993 : vidéo). Ce fut dans cette singularité, plus que dans le formalisme des architectes cariocas et l'ambition modernisant du pays, qu'elle vit le potentiel développement de ses concepts et de sa pratique pour une esthétique brésilienne.

\section{La marge au centre}

Dès son arrivée, Lina Bo Bardi en vint à occuper une position polémique dans l'espace quasi désert de la critique architecturale au Brésil. Pensant à un futur en Amérique, elle tenta bientôt de se faire connaître comme architecte et éditrice, malgré ne pas avoir attiré l'intérêt des célèbres architectes cariocas qui haussaient ses épaules de son idéologie humaniste et sa pesante expérience européenne. En février 1947, elle signa son premier article dans une publication brésilienne. Son essai, intitulé « Na Europa, a casa do homem ruiu » (En Europe, la maison de l'homme s'est effondrée) sortit dans Rio, un magazine mensuel, détenu par un autre magnat de presse, Roberto Marinho, concourant de Chateaubriand. Bo Bardi se présenta comme chef des éditions de Domus (un poste dorénavant occupé par Ernesto Nathan Rogers), cherchant à s'établir comme une spécialiste indépendante et distinguée, surtout dans un nouveau pays où le 
chauvinisme professionnel et le machisme était aussi répandus que dans son Italie natale.

Avec grand idéalisme, elle souligna, dans son article, que "alors que les bombes démoulaient sans pitié les œuvres humaines, nous comprîmes qu'une maison doit exister pour la vie humaine" (BO BARDI, 1947 : 53). Elle rappela aux brésiliens que dans l'Europe de l'après-guerre, « pour la première fois, les gens pensent aux êtres humains, à reconstruire pour les êtres humains » (BO BARDI, 1947 : 53). Selon son avis, la guerre avait détruit le mythe de monuments urbains et, également, la monumentalité des intérieurs bourgeois. « En Europe, la reconstruction est en cours ", dit-elle, " et les maisons sont simples, clairs et modeste "(BO BARDI, 1947: 54), suggérant que l'européens «étaient gênés par leurs vieilles maisons» (BO BARDI, 1947: 54), comme s'ils avaient exposé publiquement leurs faiblesses et vices intimes. Malgré la manque de répercussion de ses idées, Bo Bardi introduit avec cet article-publié durant les fêtes de carnaval-une perspective différente aux éminents architectes brésiliens. Surtout, elle mit en place le ton de sa quête permanente dans le travail qu'elle développerait graduellement comme designer, architecte et commissaire d'expositions au Brésil.

Fig. 2. Couverture de la revue Habitat 8, éditée par Lina Bo et Pietro Maria Bardi entre 1950 et 1954 et associé aux programmes culturels et pédagogiques du Musée d'Art de São Paulo

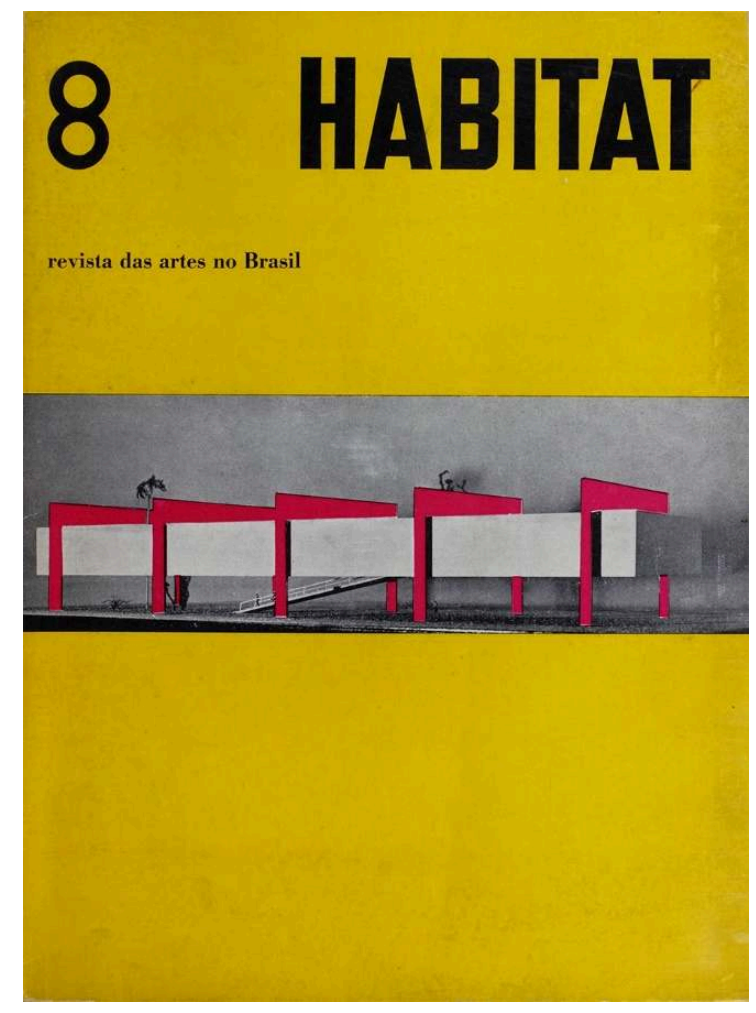

Collection Zeuler R. Lima

Quelques ans plus tard, désormais à São Paulo et occupée avec la création du MASP, Bo Bardi constata les fissures du projet moderniste brésilien, et les signala dans son travail éditorial pour Habitat (la revue associée au musée), entre 1950 et 1954- la période durant laquelle Bo Bardi devint citoyenne brésilienne et acheva son premier projet, connu comme la «maison de verre ». Dans la seconde édition du magazine, au début de 1951 elle signa un essai incisif sur l'architecture brésilienne en réponse à la critique 
internationale. Dans l'article intitulé de manière suggestive "Bela criança " (Belle enfant), elle se montrait consciente de ce que "l'architecture brésilienne a de nombreux défauts, elle est jeune, elle n'a pas eu beaucoup de temps pour faire une pause et réfléchir, elle est née soudainement comme une belle enfant " (BO BARDI, 1951:3). Elle s'accordait avec la critique étrangère sur le fait que «les brises soleil et les azulejos sont les éléments d'un vocabulaire international, que certaines formes d'Oscar [Niemeyer] sont des complaisances plastiques, que la réalisation n'est pas toujours satisfaisante » (BO BARDI, $1951: 3$ ). Elle niait, cependant, la suggestion selon laquelle "l'architecture brésilienne prenait déjà le chemin d'une académie » (BO BARDI, 1951 : 3). Elle contredisait, en même temps, les architectes néo-colonialistes en soutenant que "l'architecture contemporaine brésilienne ne découlait pas de l'architecture des jésuites ", mais de l'expression populaire, qui «possède, dans son extraordinaire volonté de faire, une fierté et une poésie [...] qui ne connaît rien des grandes villes civilisées [...] et qui n'a rien hérité des millénaires passés » (BO BARDI, 1951 : 3).

Bo Bardi soutenait que « cette capacité à prendre et à transformer sans se soucier de rien, est la force de l'architecture brésilienne » (BO BARDI, 1951: 3). Dans cet article, elle rappelle les prémisses essentiels qui en vinrent à guider ses choix intellectuels et professionnels jusqu'à la fin de sa vie au Brésil. Elle unit, dans sa pratique architecturale hybride, les principes rationalistes et héroïques du Mouvement Moderne avec une sensibilité aux répertoires locaux et populaires, dans une posture qui se tinta de romantisme révolutionnaire à la fin des années 1950 et au début des années 1960 .

Elle soutenait que, dans la marge, dans l'œuvre mineure, se trouvait l'opportunité d'une fusion et d'une révision du langage de l'architecture brésilienne. Elle poursuivit la publication d'articles dans lesquels elle analysait les maisons populaires et rurales des alentours de São Paulo et de l'intérieur du Brésil et, en 1954, elle présenta, dans le n 10 de la revue Habitat, une brève contribution non signée, intitulée "Le peuple est l'architecte ». Ce texte évoquait la simplicité et l'exactitude créatrice de la privation matérielle pour étayer son argumentaire. Les aspects esthétiques et éthiques se confondaient dans son propos selon lequel «le peuple naît avec l'architecture dans le sang, parce qu'il naît avec un sens inné de la recherche de subsistance, des opportunités, des fonctions vitales" (BO BARDI, 1954: 25). Pour elle, "les idées extravagantes [...] sont interdites au peuple quand il construit sa maison", et elle achève en suggérant que "le chaume forme un toit qui n'a rien à envier aux plus belles couvertures" (BO BARDI, 1954 : 25). De l'écriture, elle en vint à la pratique en transposant de manière littérale ses idées dans ses projets de maison de la fin des années 1950 ; une décision qui, d'ailleurs, lui valut l'incrédulité de tous pendant longtemps.

\section{De la norme à sa transgression}

La publication des articles de Lina Bo Bardi promouvant la simplicité et le dépouillement architectural tomba à un moment crucial pour la critique de l'architecture moderne brésilienne. En juin 1953, le designer suisse Max Bill, qui connaissait le couple Bardi, se trouvait à São Paulo pour donner des entrevues et des conférences sur l'art et l'architecture. Le 9 juin, il donna une conférence polémique intitulée "L'architecte, l'architecture, la société », qui portait un jugement négatif sur l'architecture brésilienne, causant un grand trouble dans les milieux académiques et professionnels. Le texte présenté, publié in extenso par Bo Bardi quelques mois plus tard dans la revue Habitat, prédisait aux architectes brésiliens que « le pays courait le risque de 
sombrer dans le plus terrible académisme antisocial, en termes d'architecture moderne [...], d'architecture comme art social. » (BILL, 1953 :B).

La visite au Ministère de l'Éducation et de la Santé, dans le contexte urbain et climatique de Rio de Janeiro, avait amené Bill à réviser les propositions basées sur les idées de Le Corbusier. Il constata les excès des architectes brésiliens, comme de ceux des autres pays, les tenant pour «des erreurs, suivant une doctrine qui se révélait impraticable [au Brésil] si ce n'est au prix d'importantes corrections» (BILL, 1953: B). Sa critique des abus de la norme moderniste se poursuivait avec l'argument selon lequel " au début, [les édifices] étaient droits et, maintenant, ils commencent à prendre les formes les plus baroques. Dans un premier temps, on a l'impression qu'il s'agit d'une construction habile, mais cela ne va pas au-delà du purement décoratif » (BILL, 1953: B). En accord avec la valorisation romantique du populaire et du vernaculaire, Max Bill demandait «pourquoi ne ri-t-on pas de la construction des précurseurs dans leur pays?» La réponse était catégorique: "C'est simplement parce que l'architecte et son commanditaire n'avaient pu résister à la tentation d'un immeuble spectaculaire, alors que l'autre, le précurseur, fait un immeuble plus conforme à son usage » (BILL, 1953 : B). Pour lui, au-delà de la création de formes, la fonction de l'architecte, dans la société moderne, serait de "rendre l'environnement de l'homme habitable et harmonieux " (BILL, 1953: B). Bo Bardi était pleinement d'accord avec lui sur ces points.

31 Max Bill concluait en attirant l'attention sur « le danger d'une conception très individualiste de la doctrine architecturale », et affirmait qu' "au Brésil, existent les forces suffisantes pour libérer l'architecture des principes académiques, des principes superflus qui n'ont aucune valeur dans ce pays » (BILL, 1953: B). La critique de l'artiste suisse fut reprise par d'autres architectes de prestige international, comme Walter Gropius, qui avait visité le Brésil, ou Ernesto Nathan Rogers, dont Bo Bardi avait fait la connaissance lors de son séjour, durant la guerre, à Milan. L'ensemble fut publié sous le titre "Report on Brazil» (Dossier sur le Brésil), par la revue nord-américaine Architectural Review, un an plus tard, en 1954, à l'apogée de la modernisation nationale et à la veille de l'élection présidentielle de Juscelino Kubitschek. Le texte - aussi que la conférence de Max Bill fut très mal reçu et connut une grande répercussion parmi les professionnels brésiliens, mais il suscita peu de réponses substantielles, à l'exception de Lucio Costa, qui discréditait la critique de Bill en notant qu'il n'était pas un architecte de formation et qu'il était arrivé au Brésil empli de préjugés (COSTA, 1954 ; 2003 : 181). Cependant, la polémique eut un bien plus grand impact sur la trajectoire d'Oscar Niemeyer, dont le langage, dès ce moment, connut une sensible reformulation et une simplification formelle et constructive. De plus, il en vint à réfléchir et à écrire systématiquement sur l'architecture moderne.

32 Niemeyer avait, initialement, été invité par Lina Bo Bardi pour créer avec elle une nouvelle revue, Brasiliana, mais il finit par publier la revue Modulo, seul, à partir de 1955. Dans un article intitulé "Le problème social en architecture ", publié en 1956, il reconnaissait l'insatisfaction croissante des architectes brésiliens, de la même manière que la réaction des deux groupes principaux : l'un «constitué de ceux qui, impressionnés par les théories traditionalistes, militent ardemment pour une architecture fondée sur la tradition et la culture de notre peuple », l'autre groupe serait celui de " ceux qui s'alarment de la pauvreté de nos constructions modernes et qui réclament des solutions plus simples et plus rationnelles » (NIEMEYER, 1954; 2003 : 184). Il avouait que «la grande majorité de nos constructions présente un bas niveau architectural ». Cependant, il choisit de continuer « $\grave{a}$ 
réfléchir à partir des systèmes de construction en usage » et de ne pas suivre les suggestions des critiques internationaux pour ce qu'il interprétait comme "une architecture plus rigide et froide - de tendance européenne", se refusant également à "plaider pour une architecture sociale » (NIEMEYER, $1954 ; 2003$ : 185).

Niemeyer croyait que ces voies mèneraient seulement à l'appauvrissement de "notre architecture dans ce qu'elle a de nouveau et de créateur, ou à la présenter de manière trompeuse, artificielle et démagogique » (NIEMEYER, 1954; 2003 : 186). Il se défendait en retournant la critique vers l'incurie des administrations publiques qui toléraient que "l'action nocive du commerce immobilier " défigure les villes brésiliennes comme une sorte de "maladie de croissance " (NIEMEYER, 1954; 2003 : 187). Dans cette position ambiguë et astucieuse, le problème de l'architecture brésilienne serait d'ordre social et non pas celui des architectes "limités que nous sommes à une architecture de classe, dans laquelle manque la base sociale nécessaire, produisant ainsi les déficiences principales »(NIEMEYER, $1954 ; 2003$ : 187).

\section{Une autre architecture}

34 Le projet de modernisation industrielle à caractère nationaliste, promu par la présidence de Juscelino Kubitschek, inaugura une nouvelle phase de modernisation dans l'architecture brésilienne. Ce développement se consolida entre les années 1950 et 1970, Oscar Niemeyer ayant maintenu sa position de chef messianique et imposé la rhétorique exubérante de la modernisation avec la création de la nouvelle capitale, Brasilia, même si, après cela, les grandes structures en béton brut émergentes à São Paulo sous aurait déstabilisé le panorama de l'architecture brésilienne. Lina Bo Bardi connut une trajectoire différente, cherchant la voie de la simplification, du "pauvre ", selon ses termes, mais non pas de l'appauvrissement. Cette option fut intimement liée à ce qu'elle avait quitté, pour des longs séjours entre 1958 et 1964, São Paulo pour Salvador de Bahia, l'ancienne capitale coloniale.

$\mathrm{Au}$ début de l'année 1958, elle fut invitée par Diógenes Rebouças à donner des conférences sur l'architecture à l'Ecole de Beaux-Arts de Salvador et, deux ans plus tard par le gouverneur de l'état de Bahia, à diriger le Musée d'Art Moderne, alors que la ville connaissait un grand développement économique et culturel grâce à l'émergence d'une industrie pétrolifère locale. Dans son effort pour affirmer son identité d'architecte brésilienne, Bo Bardi prit ses distances avec le débat fonctionnaliste qui impliquait essentiellement un axe entre Rio de Janeiro et São Paulo. Elle se rapprocha des mouvements de modernisation culturelle de la région Nordeste du pays, qui se nourrissaient des questions soulevées par la création de la SUDENE (Superintendance pour le Développement du Nordeste) et de l'ISEB (Institut des Études Brésiliennes), sous le mandat de Kubitschek. Durant la période qu'elle passa à Bahia, Bo Bardi fréquenta les intellectuels et artistes, comme le metteur-en-scène Eros Martim Gonçalves, le compositeur Hans Joachim Koellreuter, le sculpteur Mário Cravo, et le cinéaste Glauber Rocha, et plus, à Recife, des artistes et militants reliés aux mouvements de culture populaire au Nordeste et principalement au Recife. Cette proximité fut l'occasion d'une maturation et d'une radicalisation de ses positions relatives à l'architecture et au design, au Brésil. Elle continua à défendre une position intellectuelle moderne, scientifique et rationaliste, ouverte aux œuvres mineures et à l'habileté créative des exclus, cette adéquation constituant la pierre de touche de son projet pour une 
esthétique brésilienne en ce moment. Cette esthétique se fondait moins sur les principes de la modernité industrielle que sur ce qu'elle appelait le "pré-artisanat », en considérant que le Brésil n'avait pas la tradition artisanale des corporations italiennes.

Elle voyait dans la spontanéité inhérente à ceux qui demeuraient en marge de la modernisation promue par les élites brésiliennes le potentiel véritable, capable d'inverser la direction et la signification de la modernité artistique et architecturale du pays. L'architecture devrait être pauvre, non pas dans le sens économique traditionnel, mais dans celui du manque matériel et de la simplification des moyens. Bo Bardi répétait souvent que "l'homme du peuple sait construire, il est architecte par intuition, il ne se trompe pas quand il construit une maison, et construit afin de pourvoir aux nécessités de la vie » (BO BARDI, 1951: 5). Dans les conditions d'inaccessibilité aux normes modernes, "l'harmonie de sa construction est l'harmonie naturelle des choses, exempte de toute contamination par une culture artificielle, par l'orgueil et l'argent» (BO BARDI, 1951:5). Cette proposition tenait aussi bien de la politique et de la vie en société que de la phénoménologie et de l'expérience vécue. Le concept d'une architecture simple et humanisée se fondait sur la nécessité de reconnaître que «la fonction de l'architecte est, avant tout, de connaitre le mode de vie du peuple dans ses maison et de chercher les moyens techniques de résoudre ce qui rend la vie de milliards de personnes difficile » (BO BARDI, $1952:$ 3).

Cette conception en revenait aux réactions qu'elle, comme ses contemporains italiens, avaient eu face au contexte existentiel de la Seconde Guerre Mondiale. Elle tirait de son expérience éditoriale les prémisses, négligées par plusieurs ses collègues brésiliens, selon lesquels "pour un architecte, l'essentiel n'est pas de construire bien, mais de savoir comment vivent la majorité des gens" (BO BARDI, 1952: 3). Cependant le caractère progressiste et problématique de la modernisation ainsi que la recherche d'un nouvel humanisme dans la définition de son architecture pauvre n'évitaient pas une mythification romantique de la réalité ainsi qu'une emphase picturale de son langage architectural. Cette sensibilité ambivalente et hybride, esthétique et humaniste à la fois, trouva son expression dans quelques de ses projets expérimentaux réalisés à la fin des années 1950 et dans la décennie suivante, projets qui alimentèrent son travail ultérieur. Se détachent de cet ensemble deux résidences de classe moyenne et deux musées à São Paulo et à Salvador. 
Fig. 3. Maison Valéria Cirell, projetée par Lina Bo Bardi et construite à São Paulo (1957-59)

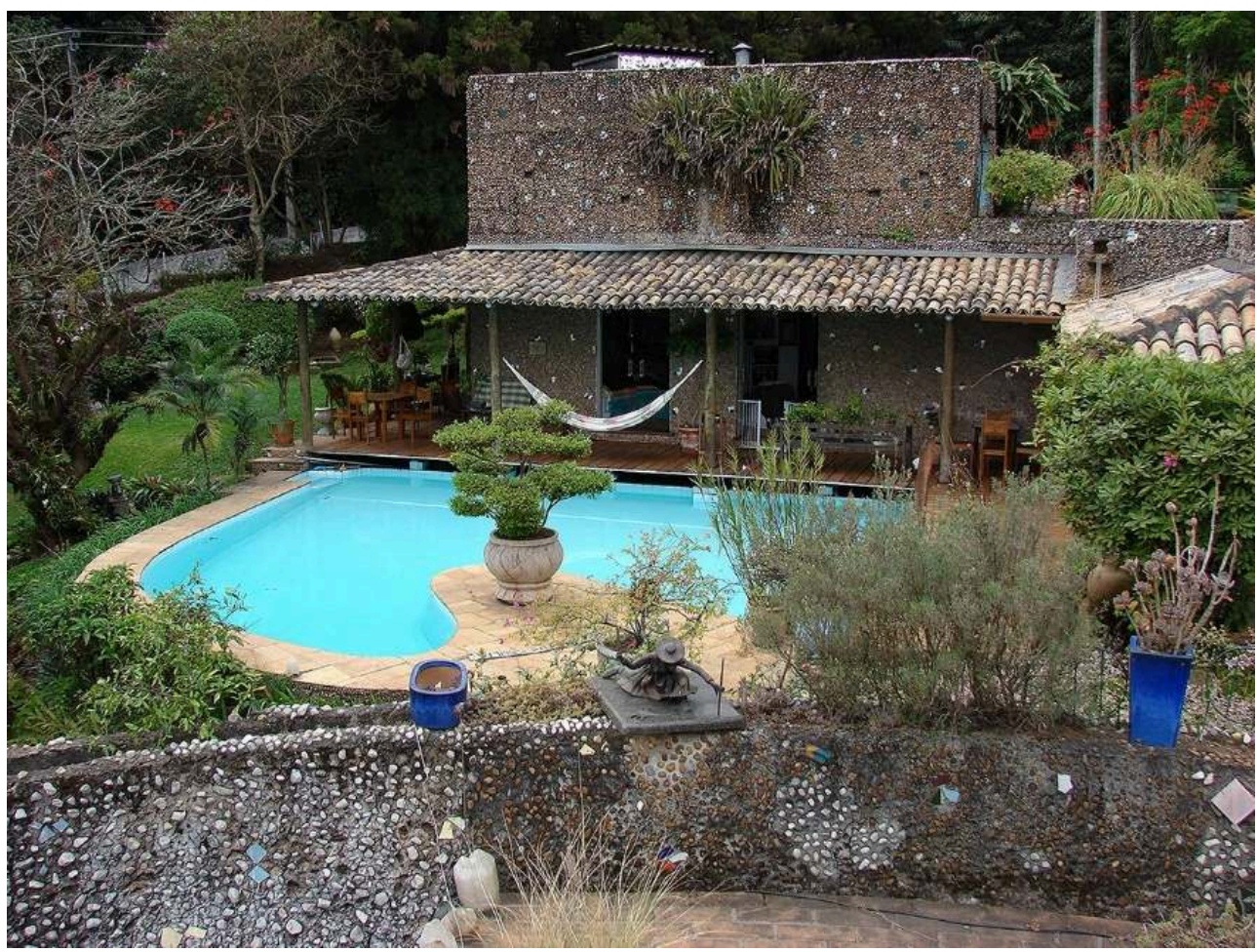

Photo : Zeuler R. Lima

Les deux maisons révèlent l'intérêt de l'architecte pour le développement d'un langage de la construction fondé sur la simplification des moyens et des matériaux, bien que, au début, l'essentiel de cette simplification ait été visuelle. La maison Valéria Cirell (São Paulo, 1957-59) incorpore des éléments du paysage et de l'architecture rurale et vernaculaire, comme par exemple l'usage des vérandas en chaume et de structures rustiques de troncs d'eucalyptus, alors même que le volume global de la maison se maintenait à l'intérieur de la norme formelle du cube moderne. La maison du "Chame-Chame» (Salvador 1960-64) hésitait initialement entre le même ordre volumétrique et les éléments organiques, se résolvant à la fin en lignes courbes qui embrassaient un grand arbre préexistant sur le terrain. Pour les deux maisons, l'influence organique de Antoni Gaudi et de Frank Lloyd Wright est nette pour ses références aux éléments naturalistes et les murs extérieurs, dans le crépi grossier servant de base à une mosaïque irrégulière de galets, avec de petits débris d'azulejos et d'objets divers, ou de même dans l'utilisation de plantes pour minimiser la présence visuelle du volume. Dans sa tentative de créer la sensation de maisons capables de prendre place dans un autre contexte culturel - déficient, mais essentiellement naïf Bo Bardi théâtralisa sa négociation avec le rationalisme moderniste. 
Fig. 4. L'ensemble colonial du Solar do Unhão, récupéré et converti dans le Musée d'Art Populair par Lina Bo Bardi à Salvador (1961-63)

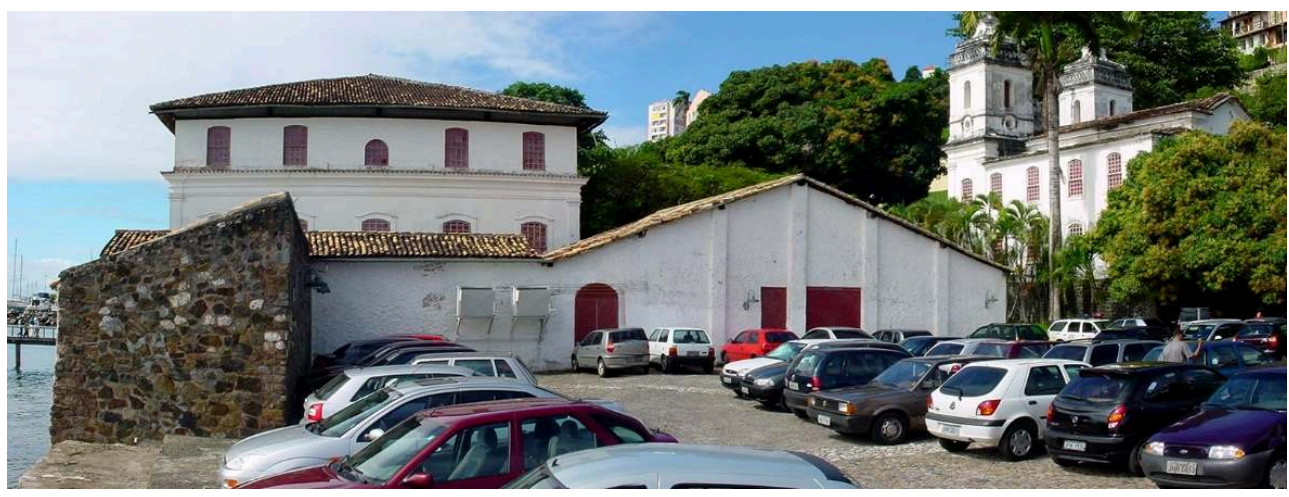

Photo : Zeuler R. Lima

Fig. 5. Escalier hélicoïdal dans l'ensemble colonial du Solar do Unhão, projeté par Lina Bo Bardi à avec références a des techniques constructives et objets vernaculaires (1963)

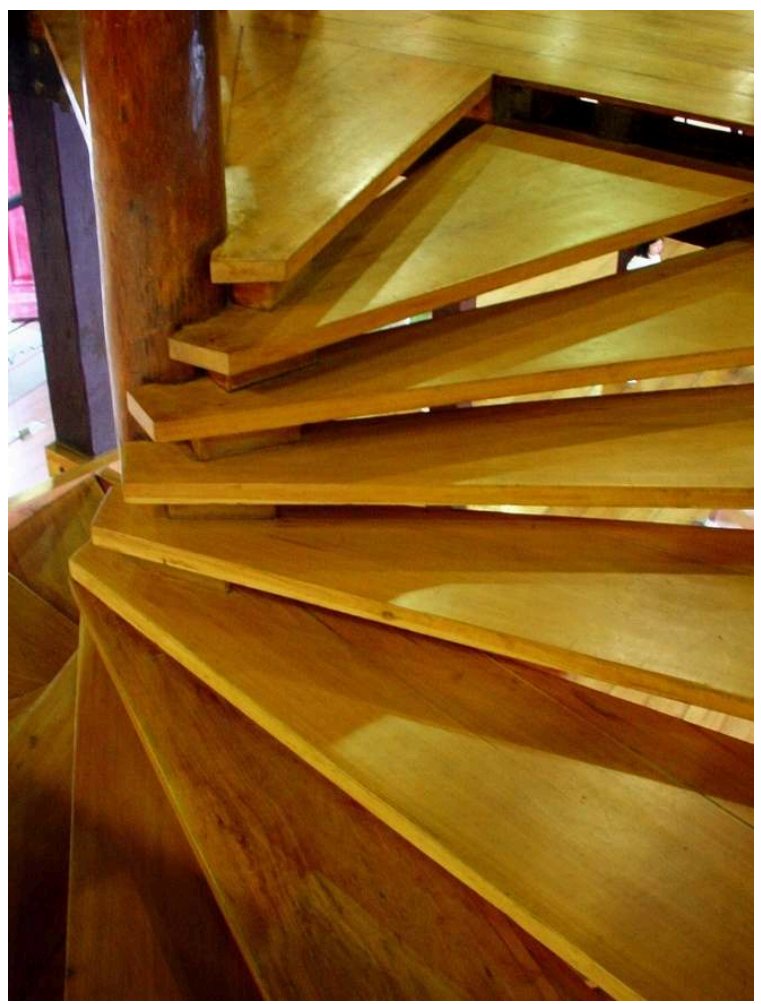

Photo : Zeuler R. Lima

Les deux musées, à savoir le siège permanent du Musée d'Art de São Paulo, sur l'avenue Paulista, et le Musée de l'Art Populaire, dans le Solar do Unhão, en bord de mer, à Salvador, furent projetés et conçus ensemble. Le projet pauliste, interrompu de nombreuses fois à la suite de problèmes financiers et politiques, prit plus de retard et ne vit le jour qu'en 1968, malgré le départ du projet en 1957, alors que celui de Salvador connut une conception plus rapide, entre 1961 et 1963, mais non moins intense. Sans prévoir le prolongement dramatique du coup d'état militaire de 1964, Bo Bardi se consacra de manière exclusive au projet du Musée d'Art Populaire, développant sa proposition à partir du Musée d'Art Moderne de Bahia qu'elle venait diriger quelques 
années auparavant. Elle pensait qu'un musée d'art ouvert à tous devrait confronter des définitions artistiques et architecturales conventionnelles et, également, interroger les frontières entre les cultures laïque et érudite. Elle voulait créer un musée-école basé sur ses expériences italiennes mais aussi sur sa collaboration avec Martim Gonçalves à l'Ecole de Théâtre et sur le succès des Centres de Culture Populaire au Recife. Elle voulait créer une "Université Populaire, » un "Centre de Documentation sur l'Art Populaire » et un «Centre d'Etudes Techniques sur le Nordeste », orienté sur le passage du pré-artisanat à l'industrie moderne (BO BARDI, 1963b : 1). Sa proposition adaptait de façon critique le modèle de Max Bill pour l'école de design d'Ulm au contexte culturel et politique effervescent du Brésil et, notamment de la Bahia d'après Kubitschek.

Le terme "pré-artisanat » permettait de contourner les difficultés de la définition traditionnelle du folklore. Lina Bo Bardi voyait dans cette expression esthétique spontanée un savoir-faire transgressif et révolutionnaire. Elle croyait que cette habileté transformatrice rendrait possible le fait qu'un peuple défavorisé, mais doté d'une sensibilité artistique intrinsèque, donnerait au Brésil une nouvelle identité culturelle. Elle choisit pour le site du centre culturel et pédagogique le vieux Solar do Unhão, un ensemble de bâtiments datant du XVI ${ }^{e}$ siècle, monumental et en très mauvais état. Il était impossible, à cause d'une documentation lacunaire, de rendre à ces bâtiments leur apparence originelle. Aussi Bo Bardi proposa-t-elle la réhabilitation des bâtiments historiques, en y ajoutant de nouveaux éléments en application de ses principes conceptuels. Parmi les éléments les plus significatifs de ce travail, on retiendra une vaste terrasse au bord de l'eau et la création d'un escalier de bois central donnant sur l'espace du Solar dédié aux expositions. Pour cet escalier, à la fois moderne et enraciné dans la culture populaire du Sertão, les tenons des marches sont en bois travaillé selon les techniques traditionnelles pour fabriquer des chars à bœuf.

41 Le musée fut ouvert au public en 1963 avec l'exposition «Nordeste », qu'elle avait organisée avec l'aide des artistes et anthropologues de la région. L'exposition proposait une collection d'objets populaires - des ustensiles de cuisine, des jouets ou des armes qu'elle appelait "objets techniques", placés sur des caisses de bois rustiques ressemblant celles venues de marchés et de foires. Le musée, comme l'exposition, rendait tangible, selon le catalogue de l'inauguration, "la recherche désespérée et positive [...] pour son droit d'exister [...] une affirmation de beauté obtenue avec la précision que seul l'affrontement au réel peut produire » (BO BARDI, 1963: 1). Ce matériel représentait métaphoriquement, pour Bo Bardi, «ce que la civilisation moderne considérait comme du déchet ». Elle concevait l'exposition comme une "accusation dépourvue de toute timidité, qui conteste les conditions dégradantes imposées à ceux qui faisaient un effort désespéré pour produire une culture " (BO BARDI, 1963: 1). Quelques mois après l'ouverture, le coup d'état militaire imposa le changement de direction dans la vie politique et culturelle du pays et, indirectement, du musée, modifiant ainsi ses objectifs initiaux et interrompant le travail de rénovation entrepris par Bo Bardi et ses contemporains à Bahia et dans le Nordeste.

42 Après son retour définitif à São Paulo, l'architecte se consacra à la conclusion de l'édifice du Musée d'Art de São Paulo, le MASP, appelé à devenir l'une de ses œuvres les plus connues. Le projet, commencé en 1957, avait des caractéristiques bien différentes de celles du bâtiment fini effectivement entre 1968 et 1969. La première hypothèse, rapidement écartée, était une pyramide de verre en forme de serre, avec des plantes et une rampe hélicoïdale intérieure, analogue à celle du Musée Gugenheim de Frank Lloyd 
Wright. Bo Bardi abandonna ces premières ébauches naturalistes pour la forme austère et ondulante du Crown Hall de Mies van der Hohe, louée de manière nuancée par les modernistes brésiliens mais critiquée par Max Bill, dont elle s'était déjà servie pour l'avant-projet d'un Musée d'Art, destiné à la ville de São Vicente, sur le littoral pauliste, en 1951. Ce deuxième modèle, rationaliste, répondait mieux aux exigences de la législation qui interdisait toute construction sur l'emplacement de l'ancien Belvédère du Trianon.

43 L'élément spécifique du projet de Lina Bo Bardi par rapport aux ouvrages des autres modernistes tenait dans le choix initial d'un volume suspendu, mais pratiquement opaque. Elle proposait un traitement des surfaces du volume selon les principes naturalistes qu'elle avait déjà conçus antérieurement, à savoir l'inachèvement apparent à la manière de Gaudi, avec des cailloux, tessons de céramique et plantes. Peu à peu, pendant les longues années de conception du projet, à cause de problèmes techniques, émergea l'idée d'un volume complètement transparent, à l'image de bien d'autres bâtiments modernistes, au Brésil. Le résultat est d'une conception formelle simple, à une échelle spectaculaire, d'une apparence générale brute, utilisant une portée libre en béton précontraint de 74 mètres de largeur au-dessus d'un bâtiment à demi enterré qui remplaçait l'ancien Belvédère du Trianon.

Fig. 6. Le Musée d'Art de São Paulo (MASP) à l'avenue Paulista dans sa version finale, en béton brut et verre, mais sans les éléments naturalistes initialement conçus par Lina Bo Bardi (1957-68)

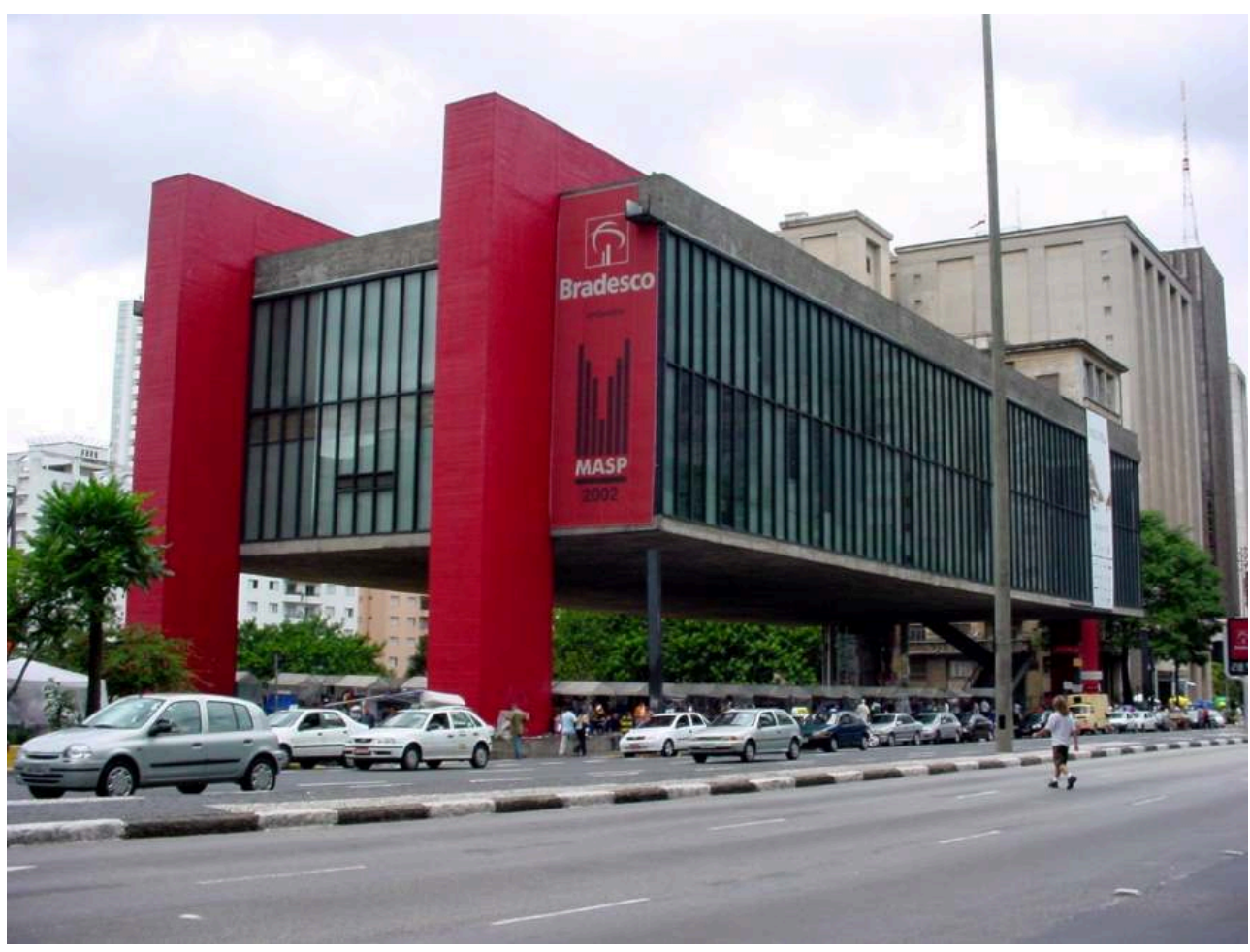

Photo : Zeuler R. Lima

L'audace structurelle du musée allait plus nettement dans le sens des projets des architectes paulistes inspirés par João Vilanova Artigas que de ceux que Lina Bo Bardi avait développés indépendamment à la fin des années 1950. Les bâtiments qu'elle avait projetés, à cette époque, avaient tenté une rénovation du langage architectural brésilien, négociant avec le rationalisme moderne grâce aux références populaires, 
organiques et vernaculaires. Le résultat final du projet du MASP, de par les concessions consenties, révisaient, ces caractères, allant jusqu'à les nier. Étant donnés la longue durée du projet et sa relation aux travaux de la même période ou postérieures, le bâtiment du MASP suggère une ambivalence, sinon un certain anachronisme interne, dans l'œuvre de Lina Bo Bardi, malgré sa signification importante pour l'architecture brésilienne. Elle tenta, cependant, toujours de mettre l'accent sur le fait qu'elle cherchait, dans le projet du musée pauliste, la simplification. En cherchant à maintenir sa cohérence rhétorique, elle réfutait toute interprétation du bâtiment comme une expression architecturale extravagante, soutenant qu'il était le produit des conditions imposées par le terrain. Sa position théorique resta constante, bien que le projet du MASP démontrât qu'on ne gagne jamais complètement dans les négociations avec le faire politique et culturel, entre le pouvoir établi et les stratégies d'insertion sociale et symbolique.

Les procédures hybrides et ambivalentes préconisées par Bo Bardi - l'économie des moyens, la résistance permanente à la norme rationaliste moderne, la retenue et l'exubérance des formes, des échelles et de la composition des projets - furent souvent revues et retravaillées dans son œuvre postérieure, entre 1960 et 1992. La notion d'architecture "pauvre ", conçue pour négocier avec la modernité, lui fut également utile dans ses confrontations avec ses contemporains européens comme brésiliens dans l'après guerre et du développentisme de la période Kubitschek. Dans cette notion, des différentes visions $\mathrm{du}$ monde coexistaient, faisant de tout objet un hybride aux frontières conceptuelles poreuses. Ses projets, en effet, ne se dissociaient pas de son expérience vécue, ou de son travail intellectuel et créatif. Sa condition au tant que femme et étrangère et sa posture critique en regard de la modernisation au Brésil introduisirent des réalités moins visibles dans son projet esthétique et politique, notamment à partir de la fin des années 1950.

Tant qu'une architecte qui se disait antiféministe, une européenne en opposition à l'ethnocentrisme, Bo Bardi défendit la vision d'une singularité du projet moderniste tenu non plus comme une condition isolée et réifiée mais bien comme une altérité produite par la différence entre les identités culturelles. Son travail d'érosion et, simultanément, de construction de nouvelles normes et valeurs, prit place dans un contexte historique, géographique et culturel propice et son encore ouvert à être explorer. Sa double appartenance aux deux côtés de l'Atlantique ainsi que son héritage culturel multiple et son transit dans un environnent traditionnellement masculin lui conférèrent une position privilégiée pour penser et agir. A la fin de sa vie, lors d'une entrevue sur son projet pour le MASP, elle remarqua que "les spécialistes ne l'ont pas aimé, mais le peuple, oui. » Et, en conclusion et avec une pointe d'ironie, elle ajouta: "Savez-vous qui a fait ça ? C'était une femme ». (BO BARDI, 1993 : vidéo).

\section{BIBLIOGRAPHIE}

BARDI, Pietro Maria. Sodalício com Assis Chateaubriand. MASP, São Paulo, 1982. 14. 
BILL, Max. «O arquiteto, a arquitetura, a sociedade», dans Habitat, $\mathrm{n}^{\circ} 12$, septembre 1953, A et B. BO, Lina. «Recensioni: Un importante libro sull'architettura», dans Lo Stile $n^{\circ} 10$, octobre 1941, 34.

BO BARDI, Lina. « Na Europa, a casa do homem ruiu », dans Rio n 92, février, 1947, 53-55 et 95. BO BARDI, Lina. «Amazonas: O povo arquiteto», dans Habitat n 1, octobre-décembre 1950, 25. BO BARDI, Lina. «Bela criança», dans Habitat n² 2, janvier-mars 1951, 3.

BO BARDI, Lina. «Construir é viver» Habitat n 7, São Paulo, janvier-mars 1952, p. 3

BO BARDI, Lina. «O povo é arquiteto», dans Habitat n 10, janvier-mars 1954, 18.

BO BARDI, Lina. «Curriculum vitae», transcription, 1957. ILBPMB, São Paulo.

BO BARDI, Lina. Solar do Unhão. Governo da Bahia, Salvador 1963b. Manuscrit décrivant la récupération, la revitalisation et la proposition pour le Solar do Unhão à Salvador. ILBPMB, São Paulo.

BO BARDI, Lina. Nordeste, catalogue d'exposition, Salvador, novembre 1963a. ILBPMB, São Paulo. BO BARDI, Lina. Conférence à la FAUUSP, 14 avril 1989. Instituto Lina Bo e Pietro Maria Bardi (ILBPMB), São Paulo, transcription, 5.

BO BARDI, Lina. Entrevue avec Silvio Giannini, « Mostra reúne pela primeira vez obra de Lina Bo Bardi », dans Folha de São Paulo, 10 avril 1989. ILBPMB, São Paulo.

BO BARDI, Lina. Entrevue avec Fábio Malavoglia, Centro Cultural São Paulo, 10 mars 1991, transcription. ILBPMB, São Paulo.

BRUNETTI, Fabrizio. L'architettura in Italia negli anni della ricostruzione. Alinea, Florence, 1998. COSTA, Lucio. «Oportunidade perdida» (1954), dans Alberto Xavier (org.), Depoimento de uma geração. Cosac \& Naif, São Paulo, 2003,181-184.

DE GRAZIA, Victoria. Le donne nel regime fascista. Marsilio, Venice,1993.

FASOLO, Vicenzo. La Facoltà di Architettura di Roma (nel suo trentacinquesimo anno di vita). Facoltà di Architettura, Rome,1955.

MARTIGNONI, Massimo. Gio Ponti: Gli anni di stile, 1941-1947. Abitare Segesta, Milan, 2002.

LIMA, Zeuler. Lina Bo Bardi. Yale University Press, New Haven et Londres, 2013.

MICHILES, Aurélio et FERRAZ, Isa Grinspum (mis-en-scène). Lina Bo Bardi, vidéo documentaire, ILBPMB, São Paulo, 1993.

NIEMEYER, Oscar. «O problema social da arquitetura» (1954), dans Alberto Xavier (org.), Depoimento de uma geração. Cosac \& Naif, São Paulo, 2003, 184-187.

TENTORI, Francesco. P. M. Bardi, ILBPMB, São Paulo, 1990.

TENTORI, Francesco. Quattro architetti brasiliani e un uomo eccezionale. IUAV, Venice,1995.

ZEVI Bruno. «Idee per A» et «Per Bardi e Pagani» notes, ca. Octobre 1945. Fondazione Bruno Zevi, Rome. 


\section{RÉSUMÉS}

Lina Bo Bardi, l'architecte brésilienne d'origine italienne produit un ensemble de travaux significatifs non seulement en Amérique Latine, mais aussi dans le contexte de l'architecture de la deuxième moitié du XXème siècle. Cet article propose une introduction critique à sa pensée et son œuvre, qui offrent des exemples opportuns d'hybridation culturelle en communication avec une vie intense entre différentes conditions géographiques, historiques et sociaux, aussi que de différentes visions du monde, théories et pratiques. Cette narrative expose les opportunités, les défis et les réalisations rencontrés par une femme créatrice qui établi sa réputation dans une profession traditionnellement commandé par une élite masculine en offrant, souvent, de la résistance au courant intellectuel des idées dominantes.

\section{INDEX}

Mots-clés : Bo Bardi (Lina), architecture, Brésil, modernisme, XXème siècle

\section{AUTEUR}

\section{ZEULER R. M. DE A. LIMA}

Professeur Associé - Washington University in Saint Louis School of Design and Visual Arts. Zeuler R. M. de A. Lima est architecte, enseignant-chercheur, dessinateur, et auteur de publications sur l'art, l'architecture et l'urbanisme modernes et contemporains, y compris plusieurs études et la biographie complète sur Lina Bo Bardi (Yale University Press, automne 2013). Sa recherche lui accorda, à l'unanimité, le premier Prix International Bruno Zevi pour l'histoire et la critique de l'architecture en 2007.

zlima[at]wustl.edu

http://www.samfoxschool.wustl.edu/portfolios/faculty/zeuler_lima 